\title{
Finite-Time Consensus Algorithm for Multiple Nonholonomic Disturbed Systems with Its Application
}

\author{
Shang Shi, ${ }^{1}$ Xin Yu, ${ }^{1,2}$ and Guohai Liu' \\ ${ }^{1}$ School of Electrical and Information Engineering, Jiangsu University, Zhenjiang 212013, China \\ ${ }^{2}$ Key Laboratory of Measurement and Control of Complex Systems of Engineering, Ministry of Education, Nanjing 210096, China \\ Correspondence should be addressed to Xin Yu; yuxin218@163.com
}

Received 4 February 2015; Accepted 4 April 2015

Academic Editor: Thierry Floquet

Copyright (c) 2015 Shang Shi et al. This is an open access article distributed under the Creative Commons Attribution License, which permits unrestricted use, distribution, and reproduction in any medium, provided the original work is properly cited.

\begin{abstract}
This paper deals with the problem of finite-time consensus of multiple nonholonomic disturbed systems. To accomplish this problem, the multiple nonholonomic systems are transformed into two multiple subsystems, and these two multiple subsystems are studied, respectively. For these two multiple subsystems, the terminal sliding mode (TSM) algorithms are designed, respectively, which achieve the finite-time reaching of sliding surface. Next, a switching control strategy is proposed to guarantee the finite-time consensus of all the states for multiple nonholonomic systems with disturbances. Finally, we demonstrate the effectiveness of the proposed consensus algorithms with application to multiple nonholonomic mobile robots.
\end{abstract}

\section{Introduction}

Over the last ten years, there has been increasing interest in the control problem of multiagent systems. Driven by its application to various scientific communities, such as the control of formations, optimization, and task assignment, many results have been given in recent years (see, e.g., [1-3] and the references therein).

As one of the most fundamental and important issues, consensus problem means that all the states of agents are required to agree with a common desired value. Since the exchange of information can only occur between the agent and its neighbours, the state consensus control of multiagent systems becomes difficult and challenging. We can classify the multiagent systems into leaderless and leader-follower systems. Many consensus protocols have been proposed for the leaderless and leader-follower multiagent systems in [4-7] and the references therein. Since many practical systems can be transformed into the first-order or secondorder dynamics, it is significant and necessary to study the consensus algorithms for the first-order and second-order multiagent systems. The first-order multiagent systems with fixed or switching interconnection topology have been studied deeply in $[8,9]$ and the references therein. However, for the second-order multiagent systems, the problem becomes complicated and many existing consensus algorithms of the first-order multiagent systems can not be extended to it. In the last few years, lots of consensus algorithms have been proposed to solve the consensus problem of second-order multiagent systems (e.g., [10-12]).

Most existing consensus algorithms mentioned above for multiagent systems are usually asymptotic consensus algorithms. Since the finite-time stability appeared in [13], finite-time control has received compelling attention for its faster convergence and better robustness (e.g., [14-20]). To further investigate the control problems of multiagent systems with the property of finite-time convergence, some results have been given for the first-order and second-order multiagent systems (e.g., [21-27]). The work in [28] proposed a finite-time consensus via binary control protocols for the first-order systems which only requires sign information of the relative state measurements. In [29], the terminal sliding mode technique was used to design finite-time consensus algorithms for second-order systems. The work in [30] proposed a finite-time consensus protocol for leaderless and leader-follower second-order multiagent systems.

Most of the existing consensus algorithms were designed only for the simple single or double integrators. Since 
many existing nonholonomic systems with nonholonomic constraints can not be transformed as simple single or double integrators, such as mobile robots (see, e.g., [31]), these existing finite-time consensus algorithms are generally not applicable. There have been several results of finite-time consensus algorithms for the multiple nonholonomic systems in recent years. The paper [32] discusses the cooperative control of multiple nonholonomic agents under different communication scenarios. In [33, 34], finite-time consensus protocols are presented for nonholonomic mobile agents with and without communication delay, respectively. In paper [35], the finite-time leader-following consensus problem for multiple nonholonomic mobile agents is discussed.

It is worth noting that these papers do not consider the problem of finite-time consensus of multiple nonholonomic uncertain systems. In this paper, the main contribution is that the finite-time consensus problem for a class of multiple nonholonomic uncertain systems is solved. To address this problem, we first proposed a finite-time consensus protocol for the second-order integral systems with uncertainties, which can be seen as a more general case of that in [30]. In addition, a finite-time consensus protocol is obtained for the first-order multiple integral systems in the presence of disturbances. Then, a switched strategy is designed to guarantee that the state consensus for nonholonomic multiagent systems with disturbances can be achieved in finite time.

The rest of this paper is arranged as follows. Section 2 presents the control objective and some preliminary results. Section 3 gives the finite-time consensus protocols design for multiple nonholonomic systems with disturbances, which are the main results of the paper. Simulation results of application to multiple nonholonomic mobile robots are given in Section 4 and finally conclusion is drawn in Section 5.

\section{Problem and Preliminaries}

In this section, a basic concept about graph theory, some useful lemmas, and control objective are introduced.

2.1. Graph Theory. Consider a leaderless multiagent system. Suppose that there are $n$ nodes whose information can be exchanged with the single agent undirected neighbors. Define an undirected graph $G=\{V, E, A\}$, where $V=\{1, \ldots, n\}$ is the set of nodes, $E$ is the set of edges, and $A=\left[a_{i j}\right] \in R^{n \times n}$ is the weighted adjacency matrix of $G$ with nonnegative elements. Compared to the directed graph, the edge $(i, j)$ denotes that $i$ th and $j$ th node can obtain information from each other. If there is an edge between the $i$ th agent and the $j$ th agent, then $a_{i j}=a_{j i}>1$. We define specially $a_{i i}=0$ for all $i=1, \ldots, n$. Let $D=\operatorname{diag}\left\{d_{1}, \ldots, d_{n}\right\}$ as a diagonal matrix, where $d_{i}=$ $\sum_{j=1}^{n} a_{i j}=\sum_{j \in N_{i}} a_{i j}$. Then, the Laplacian matrix of digraph $G$ is defined as $L=D-A$. Similar to the directed graph, the undirected graph $G$ is connected if there is an undirected path between any two agents.

\subsection{Some Lemmas}

Lemma 1 (see [36]). If L is the Laplacian matrix of a connected undirected graph $G$, one has

$$
x^{T} L x=\frac{1}{2} \sum_{i, j=1}^{n} a_{i j}\left(x_{i}-x_{j}\right)^{2}=\frac{1}{2} \sum_{i=1}^{n} \sum_{j \in N_{i}} a_{i j}\left(x_{i}-x_{j}\right)^{2},
$$

for any $x=\left[x_{1}, \ldots, x_{n}\right]^{T}$. It is easy to conclude that 0 is a simple eigenvalue of $L$ whose associated eigenvector is 1 . Assume that $\lambda_{1}=0 \leq \lambda_{2} \leq \cdots \leq \lambda_{n}$ is the eigenvalues of $L$. Then one has $\lambda_{2}>0$. If $1^{T} x=0$, then $x^{T} L x \geq \lambda_{2} x^{T} x$.

The lemma below presents a Lyapunov-like theorem for finite-time stability of nonlinear systems.

Lemma 2 (see [14]). Consider the system

$$
\begin{aligned}
\dot{x} & =F(x), \\
F(0) & =0, \\
x & \in R^{n} .
\end{aligned}
$$

Suppose there is $C^{1}$ function $V(x)$ defined on a neighborhood $\widehat{U} \subset R^{n}$ of the origin. If the function $V(x)$ satisfies the following conditions where $c>0$ and $0<\alpha<1$ are real numbers,

(1) $V(x)$ is positive definite on $\widehat{U}$,

(2) $\dot{V}(x)+c V^{\alpha}(x) \leq 0, \forall x \in \widehat{U}$,

then, the origin of system is locally finite-time stable. The settling time, depending on the initial state $x(0)=x_{0}$, satisfies

$$
T_{x}\left(x_{0}\right) \leq \frac{V\left(x_{0}\right)^{1-\alpha}}{c(1-\alpha)} .
$$

If $V(x)$ is also radially unbounded and $\widehat{U}=R^{n}$, the system is globally finite-time stable.

In order to construct the finite-time controller, the following three lemmas about some useful inequalities are needed.

Lemma 3 (see [17]). For any $a \in R, b \in R$, the following inequalities hold:

$$
\begin{array}{r}
|a+b|^{q} \leq 2^{q-1}\left|a^{q}+b^{q}\right|, \\
(|a|+|b|)^{1 / q} \leq|a|^{1 / q}+|b|^{1 / q},
\end{array}
$$

when $q \geq 1$ is a constant. If $q \geq 1$ is odd, then

$$
\begin{gathered}
|a-b|^{q} \leq 2^{q-1}\left|a^{q}-b^{q}\right|, \\
\left(|a|^{q}-|b|^{q}\right) \leq q|a-b|\left|a^{q-1}+b^{q-1}\right| .
\end{gathered}
$$

Lemma 4 (see [17]). $n$ and $m$ are two positive real numbers, and $a \geq 0, b \geq 0$, and $\pi \geq 0$ are continuous functions. Then, for any constant $c_{0}>0$,

$$
\begin{aligned}
a^{n} b^{m} \pi \leq & c_{0} a^{n+m} \\
& +\frac{m}{n+m}\left[\frac{n}{c_{0}(n+m)}\right]^{n / m} b^{n+m} \pi^{(n+m) / m} .
\end{aligned}
$$


2.3. Problem Formulation. For the leaderless systems of multiple nonholonomic agents, the graph $G$ is connected and the dynamics of the $i$ th agent is presented as

$$
\begin{aligned}
& \dot{x}_{i 1}=u_{i 1}+d_{i 1}, \\
& \dot{x}_{i 2}=\left(u_{i 1}+d_{i 1}\right) x_{i 3}, \\
& \dot{x}_{i 3}=u_{i 2}+d_{i 2},
\end{aligned}
$$

where $x_{i 1}, x_{i 2}$, and $x_{i 3}$ and $u_{i 1}, u_{i 2}$ are the states and control inputs of the $i$ th nonholonomic agent, respectively, and $d_{i 1}(t)$, $d_{i 2}(t)$ are disturbances.

In this paper, finite-time consensus protocols for leaderless multiple nonholonomic agents with disturbances are designed to achieve the state consensus in finite time. To achieve this objective, the following assumption is given first.

Assumption 5. The disturbances $d_{i 1}, d_{i 2}$ are bounded with a known positive constant $d_{0}$.

\section{Main Results}

To facilitate our design, the dynamics of the $i$ th agent is divided into two cascaded subsystems, which is composed of a first-order subsystem

$$
\dot{x}_{i 1}=u_{i 1}+d_{i 1}
$$

and a second-order subsystem

$$
\begin{aligned}
& \dot{x}_{i 2}=\left(u_{i 1}+d_{i 1}\right) x_{i 3}, \\
& \dot{x}_{i 3}=u_{i 2}+d_{i 2} .
\end{aligned}
$$

We define that

$$
\begin{aligned}
& x_{1}=\left(x_{11}, \ldots, x_{n 1}\right), \\
& e_{i 1}=\sum_{j \in N_{i}} a_{i j}\left(x_{i 1}-x_{j 1}\right), \\
& x_{2}=\left(x_{12}, \ldots, x_{n 1}\right), \\
& e_{i 2}=\sum_{j \in N_{i}} a_{i j}\left(x_{i 2}-x_{j 2}\right), \\
& x_{3}=\left(x_{13}, \ldots, x_{n 3}\right) .
\end{aligned}
$$

3.1. The Design of Finite-Time Consensus Protocol for Multiple Subsystems (9). To design the finite-time consensus algorithm for the second-order multiple subsystems (9), we first choose the control $u_{i 1}$ as

$$
u_{i 1}=k_{1} \text {, }
$$

where $k_{1}>d_{0}$ is a positive constant. In this case, the multiple subsystems (9) reduce to

$$
\begin{aligned}
& \dot{x}_{i 2}=\left(k_{1}+d_{i 1}\right) x_{i 3}, \\
& \dot{x}_{i 3}=u_{i 2}+d_{i 2} .
\end{aligned}
$$

Remark 6. In [30], when $d_{i 1}=d_{i 2}=0$, a finite-time consensus algorithm is designed for multiagent systems (12). If the condition $d_{i 1}=0,\left|d_{i 2}\right|<d_{0}$ is considered, the algorithm in [30] can only guarantee that the steady-state errors of any two agents will converge to a region $Q$ about $d_{0}$. In the following theorem, when $d_{i 1}$ and $d_{i 2}$ are all bounded by a known positive constant $d_{0}$, we extend the result in [30] to achieve the finite-time consensus for multiple subsystems (12).

Now, we are ready to state our main result in this subsection.

Theorem 7. Based on Assumption 5, for the multiple subsystems (12), the finite-time consensus protocol based on nonsingular TSM control is designed as

$$
\begin{aligned}
u_{i 2}= & -k_{4} \operatorname{sgn} S_{i 2} \\
& -k_{2}\left[x_{i 3}^{p}+k_{3}^{p}\left(\sum_{j \in N_{i}} a_{i j}\left(x_{i 2}-x_{j 2}\right)\right)\right]^{(2-p) / p}
\end{aligned}
$$

with

$$
\begin{aligned}
S_{i 2} & =x_{i 3}+\int_{0}^{\tau} k_{2}\left[x_{i 3}^{p}\right. \\
& \left.+k_{3}^{p}\left(\sum_{j \in N_{i}} a_{i j}\left(x_{i 2}-x_{j 2}\right)\right)\right]^{(2-p) / p} d \tau
\end{aligned}
$$

where $1<p=p_{1} / p_{2}<2, p_{1}, p_{2}$ are positive odd integers, $k_{4}>d_{0}$ is a positive constant, and $k_{2}, k_{3}$ are two designed positive constants to be determined later. Under the finite-time consensus protocol (13), the states of all subsystems (12) can reach a consensus in a finite time.

Proof. Firstly, we prove that the manifold $S_{i 2} \equiv 0$ can be established in a finite-time $t_{1}$ with protocol (13). Then, we show that, once the ideal sliding mode $S_{i 2} \equiv 0$ is obtained, the state consensus for multiple subsystems (12) is achieved in a finite-time $t_{2}$.

Defining $U_{1}(t)=(1 / 2) \sum_{i=1}^{n} S_{i 2}^{2}$, with $u_{i 2}$ and $S_{i 2}$ given in Theorem 7 , the derivative of $U_{1}(t)$ is

$$
\begin{aligned}
\dot{U}_{1}(t) & =\sum_{i=1}^{n} S_{i 2}\left(-k_{4} \operatorname{sign} S_{i 2}+d_{i 2}\right) \\
& \leq-\left(k_{4}-d_{0}\right) \sum_{i=1}^{n}\left|S_{i 2}\right| .
\end{aligned}
$$

By Lemma 3, one gets

$$
U_{1}^{1 / 2}(t) \leq \frac{1}{\sqrt{2}} \sum_{i=1}^{n}\left|S_{i 2}\right| .
$$

For $\forall \alpha_{1} \in\left(0,\left(k_{4}-d_{0}\right) \sqrt{2}\right)$, one can verify that

$$
\dot{U}_{1}+\alpha_{1} U_{1}^{1 / 2}=\left(\frac{\alpha_{1}}{\sqrt{2}}-\left(k_{4}-d_{0}\right)\right) \sum_{i=1}^{n}\left|S_{i 2}\right| \leq 0 .
$$


By Lemma 2, we can conclude that $U_{1}$ and so the manifold $S_{i 2}$ will reach zero in a finite-time $t_{1}$ satisfying

$$
t_{1} \leq \frac{2 U_{1}^{1 / 2}(0)}{\alpha_{1}}
$$

Once $t \geq t_{1}$, we have $S_{i 2}=0$. Then system (12) will behave in an identical fashion; namely,

$$
\begin{aligned}
& \dot{x}_{i 2}=\left(k_{1}+d_{i 1}\right) x_{i 3}, \\
& \dot{x}_{i 3}=-k_{2}\left[x_{i 3}^{p}+k_{3}^{p}\left(\sum_{j \in N_{i}} a_{i j}\left(x_{i 2}-x_{j 2}\right)\right)\right]^{(2-p) / p} .
\end{aligned}
$$

Next, we prove that there exist positive constants $k_{2}, k_{3}$ such that the states of multiple systems (19) can reach a consensus in a finite-time $t_{2}$. The stability analysis of system (19) is based on that of [30], but the perturbations are not considered there. The proof is divided into two steps.

Step 1. Define a Lyapunov function $V_{0}$ as

$$
V_{0}(t)=\frac{1}{2} x_{2}^{T} L x_{2}=\frac{1}{4} \sum_{i=1}^{n} \sum_{j \in N_{i}} a_{i j}\left(x_{i 2}-x_{j 2}\right)^{2} .
$$

With Lemma 1, the derivation of $V_{0}$ along system (19) satisfies

$$
\begin{aligned}
\dot{V}_{0}(t) & =\dot{x}_{2}^{T} L x_{2} \\
& =\left(k_{1}+d_{i 1}\right) \sum_{i=1}^{n} x_{i 3}\left(\sum_{j \in N_{i}} a_{i j}\left(x_{i 2}-x_{j 2}\right)\right) .
\end{aligned}
$$

The virtual controller $x_{i 3}^{*}=-k_{3} e_{i 2}^{1 / p}$ renders

$$
\begin{aligned}
\dot{V}_{0} \leq & -\left(k_{1}-d_{0}\right) k_{3} \sum_{i=1}^{n} e_{i 2}^{(1+p) / p} \\
& +\left(k_{1}+d_{i 1}\right) \sum_{i=1}^{n} e_{i 2}\left(x_{i 3}-x_{i 3}^{*}\right),
\end{aligned}
$$

where $k_{3}$ is a positive constant to be determined later.

Step 2. To continue the proof, we construct another Lyapunov function as

$$
V(t)=V_{0}(t)+\sum_{i=1}^{n} V_{i}(t)
$$

with

$$
V_{i}(t)=\int_{x_{i 3}^{*}}^{x_{i 3}}\left(s^{p}-x_{i 3}^{* p}\right)^{2-1 / p} d s .
$$

Taking the derivative of $V(t)$, we have

$$
\dot{V}(t)=\dot{V}_{0}+\sum_{i=1}^{n} \dot{V}_{i}(t) .
$$

In the following, the terms in the right hand of (25) are discussed, respectively.

Firstly, $\sum_{i=1}^{n} \dot{V}_{i}(t)$ is estimated. Define $\xi_{i}=x_{i 3}^{p}-x_{i 3}^{* p}$; the derivative of $V_{i}(t)$ is

$$
\begin{aligned}
\dot{V}_{i}(t)= & -\left(2-\frac{1}{p}\right) \frac{d x_{i 3}^{* p}}{d t} \int_{x_{i 3}^{*}}^{x_{i 3}}\left(s^{p}-x_{i 3}^{* p}\right)^{1-1 / p} d s \\
& +\xi_{i}^{2-1 / p} \dot{x}_{i 3} .
\end{aligned}
$$

To estimate the terms in the right hand of (26), we introduce the following proposition, whose proof is given in the appendix.

Proposition 8. There exists a positive constant $c_{1}$ such that

$$
\begin{gathered}
\frac{d x_{i 3}^{* p}}{d t} \leq k_{3}^{p} c_{1} \sum_{m=1}^{n}\left|x_{m 3}\right|, \\
\left|\int_{x_{i 3}^{*}}^{x_{i 3}}\left(s^{p}-x_{i 3}^{* p}\right)^{1-1 / p} d s\right| \leq\left|x_{i 3}-x_{i 3}^{*}\right|\left|\xi_{i}\right|^{1-1 / p} .
\end{gathered}
$$

Putting (27) into (26), one has

$$
\begin{aligned}
\dot{V}_{i}(t) & \\
\leq & -\left(2-\frac{1}{p}\right) c_{1} k_{3}^{p}\left(\sum_{m=1}^{n}\left|x_{m 3}\right|\right)\left|x_{i 3}-x_{i 3}^{*}\right|\left|\xi_{i}\right|^{1-1 / p} \\
& +\xi_{i}^{2-1 / p} \dot{x}_{i 3} .
\end{aligned}
$$

Let $d=1+1 /$ p; to continue the proof, the following proposition whose proof is given in the appendix is presented.

Proposition 9. There exists a positive constant $c_{2}, c_{3}$ such that

$$
\begin{aligned}
& \left|x_{m 3}\right|\left|x_{i 3}-x_{i 3}^{*}\right|\left|\xi_{i}\right|^{1-1 / p} \\
& \quad \leq\left(k_{3}+1\right)\left|\xi_{i}\right|^{d}+c_{2}\left|\xi_{m}\right|^{d}+k_{3} c_{3}\left|e_{m 2}\right|^{d} .
\end{aligned}
$$

Substituting (29) into (28), one has

$$
\begin{aligned}
\dot{V}_{i}(t) \leq & \frac{n(2-p)\left(k_{3}+1\right) k_{3}^{p}}{p}\left|\xi_{i}\right|^{d} \\
& +\frac{c_{2}(2-p) k_{3}^{p}}{p} \sum_{m=1}^{n}\left|\xi_{m}\right|^{d} \\
& +\frac{c_{3}(2-p) k_{3}^{p+1}}{p} \sum_{m=1}^{n}\left|e_{m 2}\right|^{d}+\xi_{i}^{2-1 / p} \dot{x}_{i 3} .
\end{aligned}
$$


By (19) and the definition of $\xi_{i}$, one has $\dot{x}_{i 3}=-k_{2} \xi_{i}^{(2-p) / p}$. Then, with (30), one gets

$$
\begin{aligned}
& \sum_{i=1}^{n} \dot{V}_{i}(t) \\
& \quad \leq\left(\frac{n(2-p)\left(k_{3}+1\right) k_{3}^{p}}{p}+\frac{n c_{2}(2-p) k_{3}^{p}}{p}-k_{2}\right) \\
& \quad \cdot \sum_{i=1}^{n}\left|\xi_{i}\right|^{d}+\frac{n c_{3}(2-p) k_{3}^{p+1}}{p} \sum_{i=1}^{n}\left|e_{i 2}\right|^{d} .
\end{aligned}
$$

Secondly, $\dot{V}_{0}$ is estimated. From (22), one obtains

$$
\begin{aligned}
\dot{V}_{0} \leq & -\left(k_{1}-d_{0}\right) k_{3} \sum_{i=1}^{n} e_{i 2}^{d} \\
& +\left(k_{1}+d_{0}\right)\left|\sum_{i=1}^{n} e_{i 2}\left(x_{i 3}-x_{i 3}^{*}\right)\right| .
\end{aligned}
$$

To estimate the last term of (32), one introduces the following proposition whose proof is given in the appendix.

Proposition 10. There exists a positive constant $c_{4}$ such that

$$
\left|\sum_{i=1}^{n} e_{i 2}\left(x_{i 3}-x_{i 3}^{*}\right)\right| \leq c_{4} \sum_{i=1}^{n}\left(\left|e_{i 2}\right|^{d}+\left|\xi_{i}\right|^{d}\right) .
$$

Using Proposition 11, one has

$$
\begin{aligned}
\dot{V}_{0}(t) \leq & \left(\left(k_{1}+d_{0}\right) c_{4}-\left(k_{1}-d_{0}\right) k_{3}\right) \sum_{i=1}^{n} e_{i 2}^{d} \\
& +\left(k_{1}+d_{0}\right) c_{4} \sum_{i=1}^{n}\left|\xi_{i}\right|^{d} .
\end{aligned}
$$

Putting (31) and (34) into (25) yields

$$
\begin{aligned}
& \dot{V}(t) \leq\left(\frac{n c_{3}(2-p) k_{3}^{p+1}}{p}+\left(k_{1}+d_{0}\right) c_{4}\right. \\
&\left.-\left(k_{1}-d_{0}\right) k_{3}\right) \sum_{i=1}^{n} e_{i 2}^{d}+\left(\frac{n(2-p)\left(k_{3}+1\right) k_{3}^{p}}{p}\right. \\
&\left.+\frac{n c_{2}(2-p) k_{3}^{p}}{p}+\left(k_{1}+d_{0}\right) c_{4}-k_{2}\right) \sum_{i=1}^{n} \xi_{i}^{d} .
\end{aligned}
$$

Now, the parameters $k_{2}, k_{3}$ are determined in turn. Let $\eta$ be as any given positive constant; one first selects the parameter $k_{3}$. Define

$$
\begin{aligned}
f\left(k_{3}\right)= & \frac{n c_{3}(2-p) k_{3}^{p+1}}{p}+\left(k_{1}+d_{0}\right) c_{4} \\
& -\left(k_{1}-d_{0}\right) k_{3} \triangleq a_{1} k_{3}^{p+1}-a_{2} k_{3}+a_{3} .
\end{aligned}
$$

It is easy to conclude that, for $\forall a_{1}, a_{2}, a_{3} \in(0, \propto)$ and $p \in$ $(1,2)$, one can select $k_{3}$ large enough to satisfy

$$
\begin{aligned}
f\left(k_{3}\right)= & \frac{n c_{3}(2-p) k_{3}^{p+1}}{p}+\left(k_{1}+d_{0}\right) c_{4} \\
& -\left(k_{1}-d_{0}\right) k_{3}>\eta .
\end{aligned}
$$

Once the parameter $k_{3}$ is fixed, one can determine the parameter $k_{2}$ satisfying

$$
\begin{aligned}
k_{2}> & \eta+\frac{n(2-p)\left(k_{3}+1\right) k_{3}^{p}}{p}+\frac{n c_{2}(2-p) k_{3}^{p}}{p} \\
& +\left(k_{1}+d_{0}\right) c_{4} .
\end{aligned}
$$

With $k_{2}, k_{3}$ satisfying (38) and (37), it is easy to verify that

$$
\dot{V}(t) \leq-\eta \sum_{i=1}^{n} e_{i 2}^{d}-\eta \sum_{i=1}^{n} \xi_{i}^{d}
$$

To estimate $V(t)$, two useful propositions are introduced in the following.

Proposition 11. With $\lambda_{2}$ given in Lemma 1, the following inequality holds:

$$
\sum_{i=1}^{n} e_{i 2}^{2} \geq 2 \lambda_{2} V_{0}
$$

The proof of Proposition 11 is straightforward by Lemma 1 and thus is omitted here.

Proposition 12. The following inequality always holds:

$$
\sum_{i=1}^{n} V_{i}(t) \leq \frac{1}{(2-1 / p) k_{3}^{1+p}} \sum_{i=1}^{n}\left|\xi_{i}\right|^{2} .
$$

With Propositions 11 and 12, one gets

$$
V(t) \leq c_{5}\left(\sum_{i=1}^{n} e_{i 2}^{2}+\sum_{i=1}^{n} \xi_{i}^{2}\right)
$$

with

$$
c_{5}=\max \left\{\frac{1}{2 \lambda_{2}}, \frac{1}{(2-1 / p) k_{3}^{1+p}}\right\} \text {. }
$$

For $0<d / 2<1$, using Lemma 3, one has

$$
V^{d / 2}(t) \leq c_{5}^{d / 2}\left(\sum_{i=1}^{n} e_{i 2}^{d}+\sum_{i=1}^{n} \xi_{i}^{d}\right)
$$

Putting (39) and (44) together, for $\forall \alpha_{2} \in\left(0, \eta / c_{5}^{d / 2}\right)$, one has

$$
\dot{V}(t)+\alpha_{2} V^{d / 2}(t) \leq\left(\alpha_{2} c_{5}^{d / 2}-\eta\right) \sum_{i=1}^{n}\left(e_{i 2}^{d}+\xi_{i}^{d}\right) \leq 0 .
$$


By Lemma 2, one concludes that $V(t)$ will reach zero in a finitetime $t_{2}$ satisfying

$$
t_{2} \leq \frac{V^{1-d / 2}\left(t_{1}\right)}{\alpha_{2}(1-d / 2)} .
$$

Hence, under control (13) with parameters $k_{2}, k_{3}$ presented in (38) and (37), the state consensus for multiple subsystems (12) can be achieved in a finite-time $T_{1} \geq t_{1}+t_{2}$.

Remark 13. It is easy to know that $V(t)=0$ means $V_{0}(t)=$ $V_{i}(t)=0$. Since the graph $G$ is connected, we obtain that $x_{i 2}=x_{j 2}, x_{i 3}=x_{i 3}^{*}$, for $\forall i, j=1, \ldots, n$. It is worth noting that $x_{i 3}^{*}=0$ once $x_{i 2}=x_{j 2}$. Thus, for $\forall i, j=1, \ldots, n$, the following equations,

$$
\begin{aligned}
& x_{i 2}=x_{j 2}=x_{2}^{*}, \\
& x_{i 3}=0, \\
& u_{i 2}=0,
\end{aligned}
$$

will be established before $T_{1}$, where $x_{2}^{*}$ is a constant. Once (47) is established, it is easy to conclude that, for $\forall t \geq T_{1}$, $i=1, \ldots, n$, if $x_{i 3}=0$ is kept with the control $u_{i 2}$, the state consensus of $x_{i 2}, x_{i 3}$ is kept no matter what the control $u_{i 1}$ is taken as.

3.2. The Design of Finite-Time Consensus Protocol for Multiple Subsystems (8). Motivated by Theorem 7, for the multiple subsystems (8), the following theorem is obtained as the main result of this subsection.

Theorem 14. Based on Assumption 5, for the multiple subsystems (8), the finite-time consensus protocol based on nonsingular TSM control is designed as

$$
u_{i 1}=-k_{6}\left[\sum_{j \in N_{i}} a_{i j}\left(x_{i 1}-x_{j 1}\right)\right]^{1 / \tilde{p}}-k_{7} \operatorname{sgn} S_{i 1}
$$

with

$$
S_{i 1}=x_{i 1}+\int_{0}^{\tau} k_{6}\left[\sum_{j \in N_{i}} a_{i j}\left(x_{i 1}-x_{j 1}\right)\right]^{1 / \tilde{p}} d \tau,
$$

where $1<\tilde{p}=\tilde{p}_{1} / \tilde{p}_{2}<2, \tilde{p}_{1}, \tilde{p}_{2}$ are positive odd integers, and $k_{6}>0, k_{7}>d_{0}$ are positive constants. Under the protocol, all the states of multiple systems (8) can reach a consensus in a finite time.

Proof. The proof is divided into two steps. At the first step, we prove that the manifold $S_{i 1}$ can reach zero in a finite time with protocol (48). In the second step, we show that, once the ideal sliding mode $S_{i 1}=0$ is established, the state consensus for multiagent subsystems (8) is achieved in a finite time.

Step 1. Define a Lyapunov function $U_{2}=(1 / 2) \sum_{i=1}^{n} S_{i 1}^{2}$; taking the derivative of $U_{2}$ yields

$$
\dot{U}_{2} \leq\left(-k_{7} \operatorname{sgn} S_{i 1}+d_{i 1}\right) S_{i 1} \leq-\left(k_{7}-d_{0}\right)\left|S_{i 1}\right| .
$$

Following the same line to obtain (17), we have

$$
\dot{U}_{2}+\alpha_{3} U_{2}^{1 / 2} \leq-\left(\left(k_{7}-d_{0}\right)-\frac{\alpha_{3}}{\sqrt{2}}\right) \sum_{i=1}^{n}\left|S_{i 1}\right| \leq 0
$$

for $\forall \alpha_{3} \in\left(0, \sqrt{2}\left(k_{7}-d_{0}\right)\right)$. Then, by Lemma $2, U_{2}$ and so the manifold $S_{i 1}$ can reach zero in a finite time.

Step 2. Once the ideal sliding mode $S_{i 1}=0$ is established, multiple systems (8) will behave in an identical fashion; namely,

$$
\dot{x}_{i 1}=-k_{6}\left[\sum_{j \in N_{i}} a_{i j}\left(x_{i 1}-x_{j 1}\right)\right]^{1 / \tilde{p}}
$$

with $k_{6}>0$. In the following, we will prove that the finitetime consensus for multiple systems (52) can be achieved.

Define a Lyapunov function $U_{3}(t)$ as

$$
U_{3}(t)=\frac{1}{2} x_{1}^{T} L x_{1}=\frac{1}{4} \sum_{i=1}^{n} \sum_{j \in N_{i}} a_{i j}\left(x_{i 1}-x_{j 1}\right)^{2} .
$$

With Lemma 1 and the definition of $e_{i 1}$, the derivation of $U_{3}$ along system (52) satisfies

$$
\begin{aligned}
\dot{U}_{3}(t) & =\dot{x}_{1}^{T} L x_{1}=\sum_{i=1}^{n} \dot{x}_{i 1}\left(\sum_{j \in N_{i}} a_{i j}\left(x_{i 1}-x_{j 1}\right)\right) \\
& =-k_{6} \sum_{i=1}^{n} e_{i 1}^{\widetilde{d}},
\end{aligned}
$$

where $\widetilde{d}=1+1 / \widetilde{p}$. From Lemma 3 , one has

$$
\dot{U}_{3}(t) \leq-k_{6}\left(\sum_{i=1}^{n} e_{i 1}^{2}\right)^{\tilde{d} / 2} .
$$

Following the same line to obtain Proposition 8, by Lemma 1, we get

$$
\sum_{i=1}^{n} e_{i 1}^{2} \geq 2 \lambda_{2} U_{3}(t)
$$

Putting (55) and (56) together, we obtain that

$$
\dot{U}_{3}(t) \leq-k_{6}\left(2 \lambda_{2}\right)^{\widetilde{d} / 2} U_{3}^{\widetilde{d} / 2}(t) .
$$

Using Lemma 2, we conclude that $U_{3}$ converges to zero with a finite time; that is, the states of multiple systems (52) can reach a consensus in a finite time.

Hence, the proof of Theorem 14 is completed with the two steps above.

3.3. The Overall Design of Finite-Time Consensus Protocol for Multiple Systems (7). Based on Theorems 7 and 14, we are ready to state our main result of this subsection for multiple systems (7). 
Theorem 15. Based on Assumption 5, for the leaderless multiagent systems (7), the finite-time consensus protocol based on a switched strategy is designed as

$$
\begin{aligned}
& u_{i 1}= k_{1}, \quad t \leq T_{1}, \\
& u_{i 1}=-k_{6}\left[\sum_{j \in N_{i}} a_{i j}\left(x_{i 1}-x_{j 1}\right)\right]^{1 / \widetilde{p}}-k_{7} \operatorname{sgn} S_{i 1}, \\
& u_{i 2}=-k_{4} \operatorname{sgn} S_{i 2}>T_{1}, \\
&-k_{2}\left[x_{i 3}^{p}+k_{3}^{p}\left(\sum_{j \in N_{i}} a_{i j}\left(x_{i 2}-x_{j 2}\right)\right)\right]^{(2-p) / p}, \\
& t \leq T_{1},
\end{aligned}
$$

$$
u_{i 2}=-k_{5} \operatorname{sgn} x_{i 3}, \quad t>T_{1},
$$

where $k_{1}, k_{4}, k_{5}, k_{7}>d_{0}, k_{6}>0,1<p=p_{1} / p_{2}<2,1<$ $\widetilde{p}=\widetilde{p}_{1} / \widetilde{p}_{2}<2, p_{1}, p_{2}, \widetilde{p}_{1}, \widetilde{p}_{2}$ are positive odd integers, $k_{2}, k_{3}$ are designed parameters satisfying (38) and (37), $T_{1} \geq t_{1}+t_{2}$ are switched time, and $S_{i 1}, S_{i 2}$ are ideal sliding surface selected as (49) and (14). Under this protocol, all the states of multiple systems (7) can reach a consensus in a finite time.

Proof. From Theorems 7 and 14, it is easy to conclude that the consensus of $x_{i 2}, x_{i 3}$ can be achieved with a finite-time $T_{1}$, and when $t \geq T_{1}$, the consensus of $x_{i 1}$ can be achieved in finite time. Therefore, here we only need to prove that the consensus of $x_{i 2}, x_{i 3}$ achieved within $T_{1}$ can be kept after $t \geq T_{1}$.

From (58), when $t>T_{1}$, we have $u_{i 2}=-k_{5} \operatorname{sign} x_{i 3}$. Define a Lyapunov function $U_{4}(t)=(1 / 2) \sum_{i=1}^{n} x_{i 3}^{2}$; the derivation of $U_{4}(t)$ satisfies

$$
\dot{U}_{4}(t)=\sum_{i=1}^{n}\left(-k_{5}\left|x_{i 3}\right|+d_{i 2} x_{i 3}\right) \leq-\left(k_{5}-d_{0}\right) \sum_{i=1}^{n}\left|x_{i 3}\right| .
$$

From Lemma 3 , we get that $\forall \alpha_{4} \in\left(0, \sqrt{2}\left(k_{5}-d_{0}\right)\right)$

$$
\begin{aligned}
\dot{U}_{4}(t)+\alpha_{4} U_{4}^{1 / 2}(t) & \leq-\left(\left(k_{5}-d_{0}\right)-\frac{\alpha_{4}}{\sqrt{2}}\right) \sum_{i=1}^{n}\left|x_{i 3}\right| \\
& \leq 0 .
\end{aligned}
$$

By Lemma 2, we conclude that $U_{4}$ will converge to zero in a finite time $\bar{t} \leq U_{4}^{1 / 2}\left(x_{i 3}\left(T_{1}\right)\right) /\left(\alpha_{4} / 2\right)$. From Remark 13, we know that $x_{i 3}\left(T_{1}\right)=0$. Therefore, it can be concluded that the convergence time $\bar{t}=0$; that is, $x_{i 3}=0$ is always kept after $t>T_{1}$.

Hence, we complete the proof of Theorem 15.

\section{Simulation Results}

In this section, we consider the leaderless multiagent systems with 5 mobile robots whose schematic is shown in Figure 1. The $i$ th robot is illustrated with two driven rear wheels and a

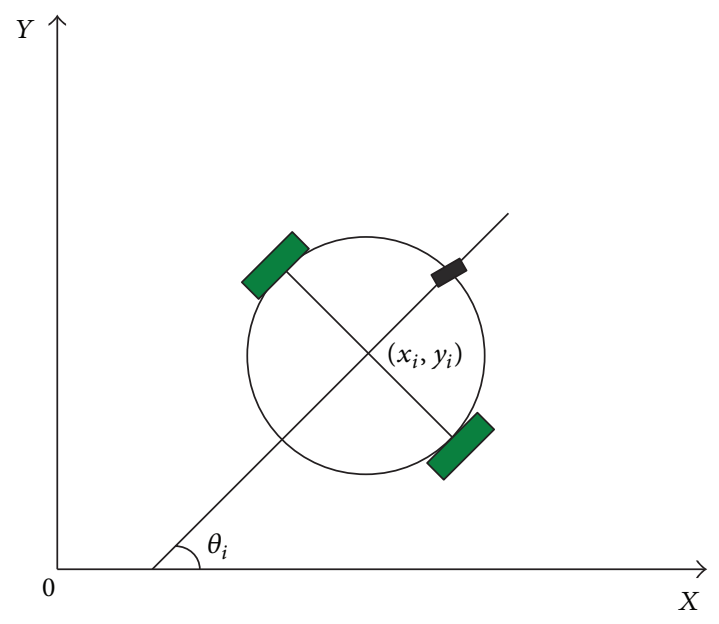

FIGURE 1: Generalized coordinates of the $i$ th car-like robot.

front freewheel. The two rear wheels are driven, respectively, with fixed orientation. The generalized coordinates are $q=$ $\left(x_{i}, y_{i}, \theta_{i}\right)$, where $x_{i}, y_{i}$ are the Cartesian coordinates of the middle point of the rear wheel and $\theta_{i}$ measures the orientation of the car body with respect to $x$-axis. Under the assumption of no wheel slips, the kinetic model of the $i$ th robot can be derived as

$$
\begin{aligned}
& \dot{x}_{i}=\left(v_{i}+d_{i 2}\right) \cos \theta_{i}, \\
& \dot{y}_{i}=\left(v_{i}+d_{i 2}\right) \sin \theta_{i}, \\
& \dot{\theta}_{i}=w_{i}+d_{i 1},
\end{aligned}
$$

where $v_{i}, w_{i}$ are the linear and angular velocities of the $i$ th mobile robot and $d_{i 1}=0, d_{i 2}=0.9 \sin (t)$ are disturbances. With the coordinate transformation

$$
\begin{aligned}
x_{i 1} & =-\theta_{i}, \\
x_{i 2} & =-x_{i} \sin \theta_{i}+y_{i} \cos \theta_{i}, \\
x_{i 3} & =x_{i} \cos \theta_{i}+y_{i} \sin \theta_{i}, \\
v_{i} & =x_{i 3} u_{i 1}+u_{i 2}=\left(x_{i} \cos \theta_{i}+y_{i} \sin \theta_{i}\right) u_{i 1}+u_{i 2}, \\
w_{i} & =-u_{i 1},
\end{aligned}
$$

system (62) is transformed as

$$
\begin{aligned}
& \dot{x}_{i 1}=u_{i 1}-d_{i 1}, \\
& \dot{x}_{i 2}=\left(u_{i 1}-d_{i 1}\right) x_{i 3}, \\
& \dot{x}_{i 3}=u_{i 2}+d_{i 2} .
\end{aligned}
$$

Then, the consensus protocols presented in Theorem 15 can be applied.

The communication among the 5 agents is given in Figure 2 . The initial states are given as $x(0)=(1,1.1,1.2,1.3,0.9)$, 


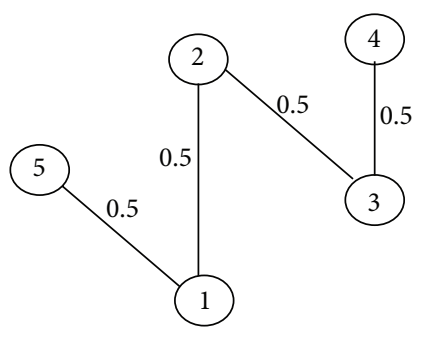

FIgURE 2: The communication among agents.

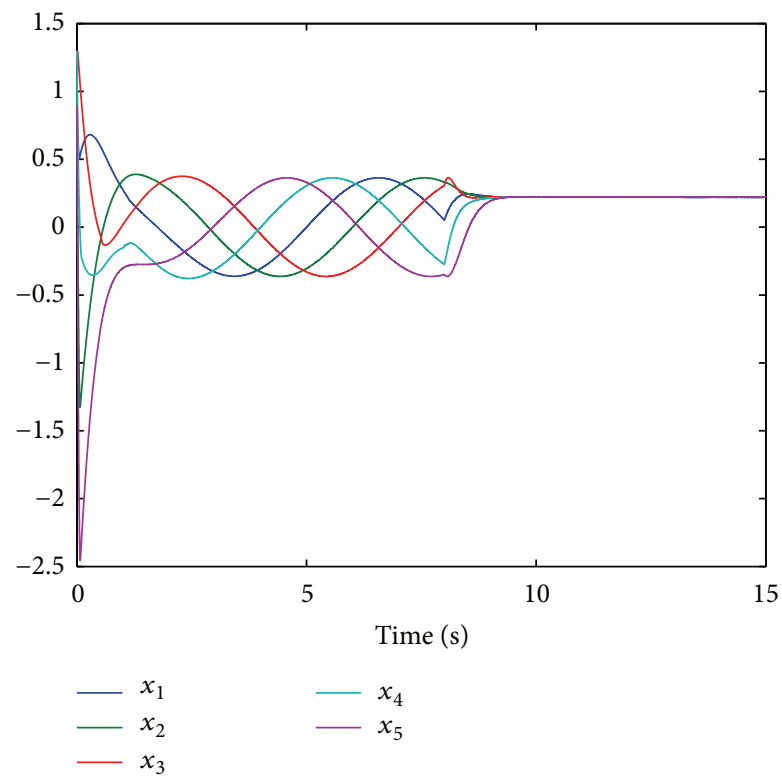

Figure 3: Agents positions $x$.

$y(0)=(1,1.1,1.2,1.3,0.9)$, and $\theta(0)=(5,6,7,4,3)$. The parameters are selected as

$$
\begin{aligned}
k_{1} & =1, \\
k_{2} & =40, \\
k_{3} & =2.7, \\
k_{4} & =1, \\
k_{5} & =1, \\
k_{6} & =2.7, \\
k_{7} & =0, \\
\eta & =1, \\
p & =\frac{9}{7}, \\
T_{1} & =10 \mathrm{~s} .
\end{aligned}
$$

Figures 3, 4, and 5 show the consensus of the positions and orientations, respectively.

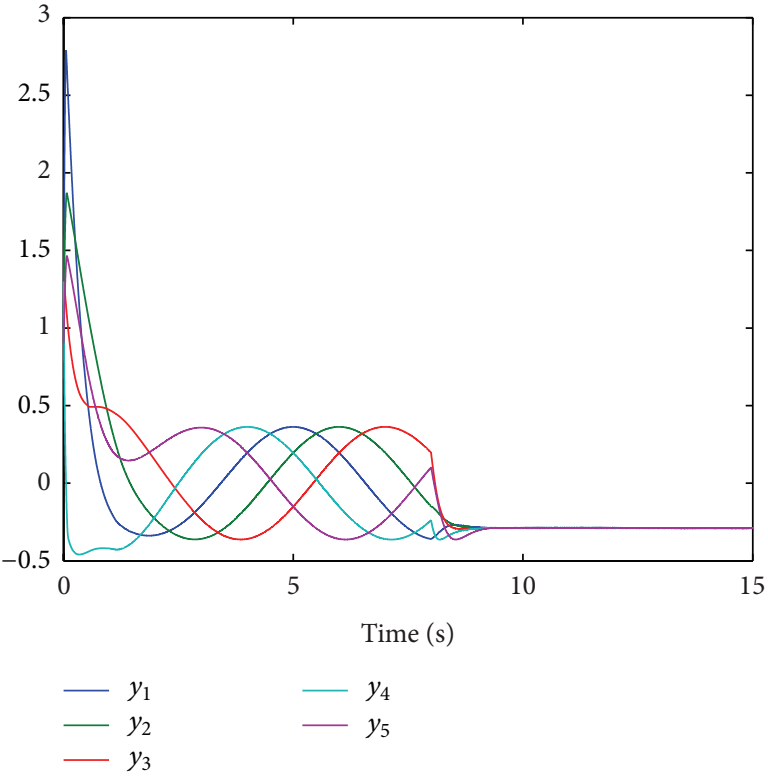

FIGURE 4: Agents positions $y$.

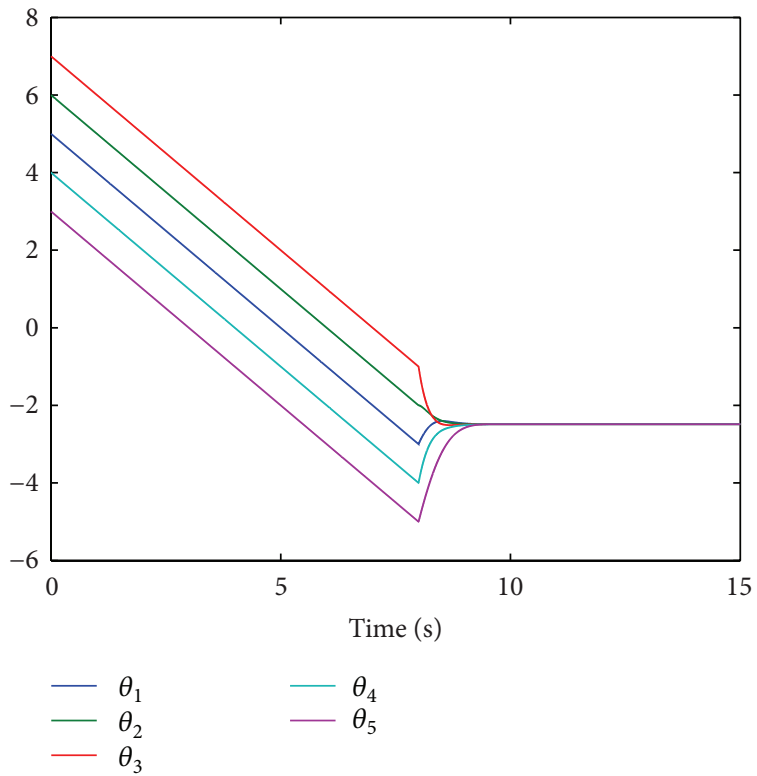

Figure 5: Agents orientation $\theta$.

\section{Conclusion}

This paper studies the problem of finite-time consensus problem of multiple nonholonomic systems in the presence of disturbances. At first, the triple chained form agent system is divided into two agent subsystems and these two agent subsystems are discussed, respectively. Then, two integral terminal sliding mode controllers are designed for the two subsystems. Finally, we demonstrate the effectiveness of the proposed consensus algorithms with application to multiple nonholonomic mobile robots. 


\section{Appendix}

Proof of Proposition 8. Define $\beta=\max _{\forall i}\left\{\sum_{j \in N_{i}} a_{i j}\right\}$ and $\gamma=$ $\max _{\forall i, j}\left\{a_{i j}\right\}$. With (13), the definition of $e_{i 2}$, and the virtual controller $x_{i 3}^{*}=-k_{3} e_{i 2}^{1 / p}$, one has

$$
\begin{aligned}
\frac{d x_{i 3}^{* p}}{d t} & =-k_{3}^{p} \dot{e}_{i 2}=-k_{3}^{p}\left(k_{1}+d_{i 1}\right) \sum_{j \in N_{i}} a_{i j}\left(x_{i 3}-x_{j 3}\right) \\
& \leq\left(k_{1}+d_{0}\right) k_{3}^{p}\left(\left|x_{i 3}\right| \sum_{j \in N_{i}} a_{i j}+\sum_{j \in N_{i}} a_{i j}\left|x_{j 3}\right|\right) \\
& \leq\left(k_{1}+d_{0}\right) k_{3}^{p}\left(\beta\left|x_{i 3}\right|+\gamma \sum_{m=1}^{n}\left|x_{m 3}\right|\right) \\
& \leq k_{3}^{p} c_{1} \sum_{i=1}^{n}\left|x_{i 3}\right|
\end{aligned}
$$

where $c_{1}$ is a positive constant.

We can also verify that

$$
\begin{aligned}
& \left|\int_{x_{i 3}^{*}}^{x_{i 3}}\left(s^{p}-x_{i 3}^{* p}\right)^{1-1 / p} d s\right| \\
& \quad \leq\left|x_{i 3}-x_{i 3}^{*}\right|\left|x_{i 3}^{p}-x_{i 3}^{* p}\right|^{1-1 / p} \\
& \quad \leq\left|x_{i 3}-x_{i 3}^{*}\right|\left|\xi_{i}\right|^{1-1 / p} .
\end{aligned}
$$

Proof of Proposition 9. With Lemma 3 and the definition of $\xi_{i}, x_{i 3}^{*}$, one has

$$
\begin{aligned}
& \left|x_{3 m}\right|\left|x_{i 3}-x_{i 3}^{*}\right|\left|\xi_{i}\right|^{1-1 / p} \\
& \quad \leq 2^{1-1 / p}\left|x_{3 m}\right|\left|\xi_{i}\right|^{1 / p}\left|\xi_{i}\right|^{1-1 / p} \\
& \quad \leq 2^{1-1 / p}\left(\left|\xi_{i}\right|\left|x_{m 3}-x_{m 3}^{*}\right|+\left|\xi_{i}\right|\left|x_{m 3}^{*}\right|\right) \\
& \quad \leq 2^{1-1 / p}\left(2^{1-1 / p}\left|\xi_{i}\right|\left|\xi_{m}\right|^{1 / p}+k_{3}\left|\xi_{i}\right|\left|e_{m 2}\right|^{1 / p}\right) \\
& \leq\left|\xi_{i}\right|^{d}+c_{2}\left|\xi_{m}\right|^{d}+k_{3}\left(\left|\xi_{i}\right|^{d}+c_{3}\left|e_{m 2}\right|^{d}\right) \\
& \quad \leq\left(k_{3}+1\right)\left|\xi_{i}\right|^{d}+c_{2}\left|\xi_{m}\right|^{d}+k_{3} c_{3}\left|e_{m 2}\right|^{d} .
\end{aligned}
$$

Proof of Proposition 10. With Lemma 3 and the definition of $\xi_{i}$, one gets

$$
\begin{aligned}
\left|\sum_{i=1}^{n} e_{i 2}\left(x_{i 3}-x_{i 3}^{*}\right)\right| & \leq \sum_{i=1}^{n}\left|e_{i 2}\right| 2^{1-1 / p}\left|x_{i 3}^{p}-x_{i 3}^{* p}\right|^{1 / p} \\
& =2^{1-1 / p} \sum_{i=1}^{n}\left|e_{i 2}\right|\left|\xi_{i}\right|^{1 / p} \\
& \leq \sum_{i=1}^{n}\left(\widetilde{c}_{4}\left|e_{i 2}\right|^{d}+\widetilde{c}_{5}\left|\xi_{i}\right|^{d}\right) \\
& \leq c_{4} \sum_{i=1}^{n}\left(\left|e_{i 2}\right|^{d}+\left|\xi_{i}\right|^{d}\right)
\end{aligned}
$$

Proof of Proposition 12. With Lemma 3, one obtains

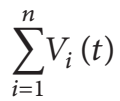

$$
\begin{aligned}
& \leq \frac{1}{(2-1 / p) 2^{1-1 / p} k_{3}^{1+p}} \sum_{i=1}^{n}\left|x_{i 3}-x_{i 3}^{*}\right|\left|\xi_{i}\right|^{2-1 / p} \\
& \leq \frac{1}{(2-1 / p) k_{3}^{1+p}} \sum_{i=1}^{n}\left|\xi_{i}\right|^{1 / p}\left|\xi_{i}\right|^{2-1 / p} \\
& =\frac{1}{(2-1 / p) k_{3}^{1+p}} \sum_{i=1}^{n}\left|\xi_{i}\right|^{2} .
\end{aligned}
$$

\section{Conflict of Interests}

The authors declare that there is no conflict of interests regarding the publication of this paper.

\section{Acknowledgments}

This work was supported by the National Nature Science Foundation (61304073), the Natural Science Foundation of Jiangsu Province (BK20130533 and BK20130536), China Postdoctoral Science Foundation (2014T70478), Specialized Research Fund for the Doctoral Program of Higher Education of China (20133227120012), Postdoctoral Fund of Jiangsu Province (1301175C), Key Laboratory of Measurement and Control of Complex Systems of Engineering, Ministry of Education (MCCSE2015A06), and Research Foundation for Advanced Talents of Jiangsu University (13JDG012). The authors express sincere gratitude to the Editor and Reviewers for their helpful comments and suggestions in improving this paper.

\section{References}

[1] W. J. Dong and J. A. Farrell, "Decentralized cooperative control of multiple nonholonomic dynamic systems with uncertainty," Automatica, vol. 45, no. 3, pp. 706-710, 2009. 
[2] W. Ren, R. W. Beard, and E. Atkins, "Information consensus in multivehicle cooperative control: collective group behavior through local interaction," IEEE Control System Magazine, vol. 27, no. 2, pp. 71-82, 2007.

[3] H. Zhang, F. L. Lewis, and A. Das, "Optimal design for synchronization of cooperative systems: state feedback, observer and output feedback," IEEE Transactions on Automatic Control, vol. 56, no. 8, pp. 1948-1952, 2011.

[4] F. C. Jiang, L. Wang, and Y. M. Jia, "Consensus in leaderless networks of high-order-integrator agents," in Proceedings of the American Control Conference (ACC '09), pp. 4458-4463, June 2009.

[5] J. H. Qin, C. B. Yu, H. J. Gao, and X. K. Wang, "Leaderless consensus control of dynamical agents under directed interaction topology," in Proceedings of the 50th IEEE Conference on Decision and Control and European Control Conference (CDCECC '11), pp. 1455-1460, December 2011.

[6] Z. K. Li, Z. S. Duan, and L. Huang, "Leader-follower consensus of multi-agent systems," in Proceedings of the American Control Conference (ACC '09), pp. 3256-3261, IEEE, St. Louis, Mo, USA, June 2009.

[7] J. Wang, Y. Tan, and I. Mareels, "Robustness analysis of leaderfollower consensus," Journal of Systems Science \& Complexity, vol. 22, no. 2, pp. 186-206, 2009.

[8] L. Moreau, "Stability of multiagent systems with timedependent communication links," IEEE Transactions on Automatic Control, vol. 50, no. 2, pp. 169-182, 2005.

[9] W. Ren and R. W. Beard, "Consensus seeking in multiagent systems under dynamically changing interaction topologies," IEEE Transactions on Automatic Control, vol. 50, no. 5, pp. 655661, 2005.

[10] Y. Hong, L. Gao, D. Cheng, and J. Hu, "Lyapunov-based approach to multiagent systems with switching jointly connected interconnection," IEEE Transactions on Automatic Control, vol. 52, no. 5, pp. 943-948, 2007.

[11] G. Hu, "Robust consensus tracking of a class of second-order multi-agent dynamic systems," Systems \& Control Letters, vol. 61, no. 1, pp. 134-142, 2012.

[12] H. Su, G. Chen, X. Wang, and Z. Lin, "Adaptive secondorder consensus of networked mobile agents with nonlinear dynamics," Automatica, vol. 47, no. 2, pp. 368-375, 2011.

[13] E. P. Ryan, "Finite-time stabilization of uncertain nonlinear planar systems," Dynamics and Control, vol. 1, no. 1, pp. 83-94, 1991.

[14] S. P. Bhat and D. S. Bernstein, "Finite-time stability of continuous autonomous systems," SIAM Journal on Control and Optimization, vol. 38, no. 3, pp. 751-766, 2000.

[15] D. Zhao and T. Zou, "A finite-time approach to formation control of multiple mobile robots with terminal sliding mode," International Journal of Systems Science, vol. 43, no. 11, pp. 19982014, 2012.

[16] S. Khoo, L. Xie, S. Zhao, and Z. Man, "Multi-surface sliding control for fast finite-time leader-follower consensus with high order SISO uncertain nonlinear agents," International Journal of Robust and Nonlinear Control, vol. 24, no. 16, pp. 2388-2404, 2014.

[17] X. Q. Huang, W. Lin, and B. Yang, "Global finite-time stabilization of a class of uncertain nonlinear systems," Automatica, vol. 41, no. 5, pp. 881-888, 2005.

[18] Y. Hong, J. Wang, and Z. Xi, "Stabilization of uncertain chained form systems within finite settling time," IEEE Transactions on Automatic Control, vol. 50, no. 9, pp. 1379-1384, 2005.
[19] T. Floquet, J.-P. Barbot, and W. Perruquetti, "Higher-order sliding mode stabilization for a class of nonholonomic perturbed systems," Automatica, vol. 39, no. 6, pp. 1077-1083, 2003.

[20] H. B. Du, C. J. Qian, M. T. Frye, and S. H. Li, "Global finite-time stabilisation using bounded feedback for a class of non-linear systems," IET Control Theory and Applications, vol. 6, no. 14, pp. 2326-2336, 2012.

[21] L. Wang and F. Xiao, "Finite-time consensus problems for networks of dynamic agents," IEEE Transactions on Automatic Control, vol. 55, no. 4, pp. 950-955, 2010.

[22] S. Yu and X. Long, "Finite-time consensus for second-order multi-agent systems with disturbances by integral sliding mode," Automatica, vol. 54, pp. 158-165, 2015.

[23] F. Xiao, L. Wang, J. Chen, and Y. Gao, "Finite-time formation control for multi-agent systems," Automatica, vol. 45, no. 11, pp. 2605-2611, 2009.

[24] C. Sun, G. Q. Hu, and L. H. Xie, "Fast finite-time consensus tracking for first-order multi-agent systems with unmodelled dynamics and disturbances," in Proceedings of the 11th IEEE International Conference on Control \& Automation (ICCA), pp. 249-254, Taichung, Taiwan, June 2014.

[25] X. Lu, R. Lu, S. Chen, and J. Lu, "Finite-time distributed tracking control formulti-agent systems with a virtual leader," IEEE Transactions on Circuits System, vol. 60, no. 2, pp. 352-362, 2013.

[26] S. H. Li and X. W. Wang, "Finite-time consensus and collision avoidance control algorithms for multiple AUVs," Automatica, vol. 49, no. 11, pp. 3359-3367, 2013.

[27] H. B. Du, Y. G. He, and Y. Y. Cheng, "Finite-time synchronization of a class of second order non-linear multi-agent systems using output feedback control," IEEE Transactions on Circuits and Systems, vol. 61, no. 6, pp. 1778-1788, 2014.

[28] G. Chen, F. L. Lewis, and L. H. Xie, "Finite-time distributed consensus via binary control protocols," Automatica, vol. 47, no. 9, pp. 1962-1968, 2011.

[29] S. Y. Khoo, L. H. Xie, and Z. H. Man, "Robust finite-time consensus tracking algorithm for multirobot systems," IEEE/ASME Transactions on Mechatronics, vol. 14, no. 2, pp. 219-228, 2009.

[30] S. Li, H. Du, and X. Lin, "Finite-time consensus algorithm for multi-agent systems with double-integrator dynamics," Automatica, vol. 47, no. 8, pp. 1706-1712, 2011.

[31] M. Defoort, T. Floquet, W. Perruquetti, and S. V. Drakunov, "Integral sliding mode control of an extended Heisenberg system," IET Control Theory \& Applications, vol. 3, no. 10, pp. 1409-1424, 2009.

[32] W. Dong and J. A. Farrell, "Cooperative control of multiple nonholonomic mobile agents," IEEE Transactions on Automatic Control, vol. 53, no. 6, pp. 1434-1448, 2008.

[33] J. Wang, Z. Qiu, and G. Zhang, "Finite-time consensus problem for multiple non-holonomic mobile agents," Kybernetika, vol. 48, no. 6, pp. 1180-1193, 2012.

[34] J. Wang, Z. Qiu, G. Zhang, and W. Yang, "Finite time consensus for multiple non-holonomic agents with communication delay," in Proceedings of the 31st Chinese Control Conference (CCC '12), pp. 6267-6272, July 2012.

[35] J. Wang, T. Gao, and G. Zhang, "Finite-time leader-following consensus for multiple non-holonomic agents," in Proceedings of the 33rd Chinese Control Conference (CCC '14), pp. 1580-1585, Nanjing, China, July 2014.

[36] R. Olfati-Saber and R. M. Murray, "Consensus problems in networks of agents with switching topology and time-delays," IEEE Transactions on Automatic Control, vol. 49, no. 9, pp. 15201533, 2004. 


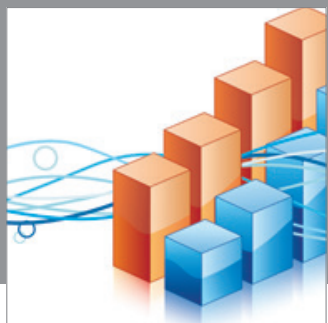

Advances in

Operations Research

mansans

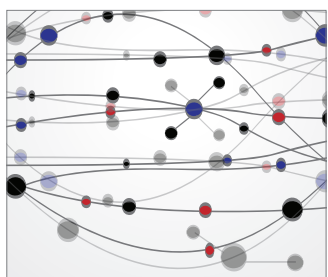

The Scientific World Journal
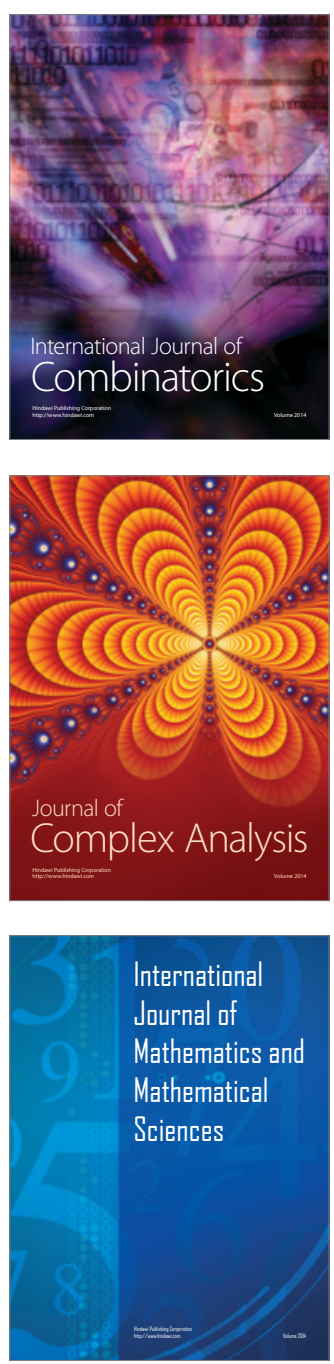
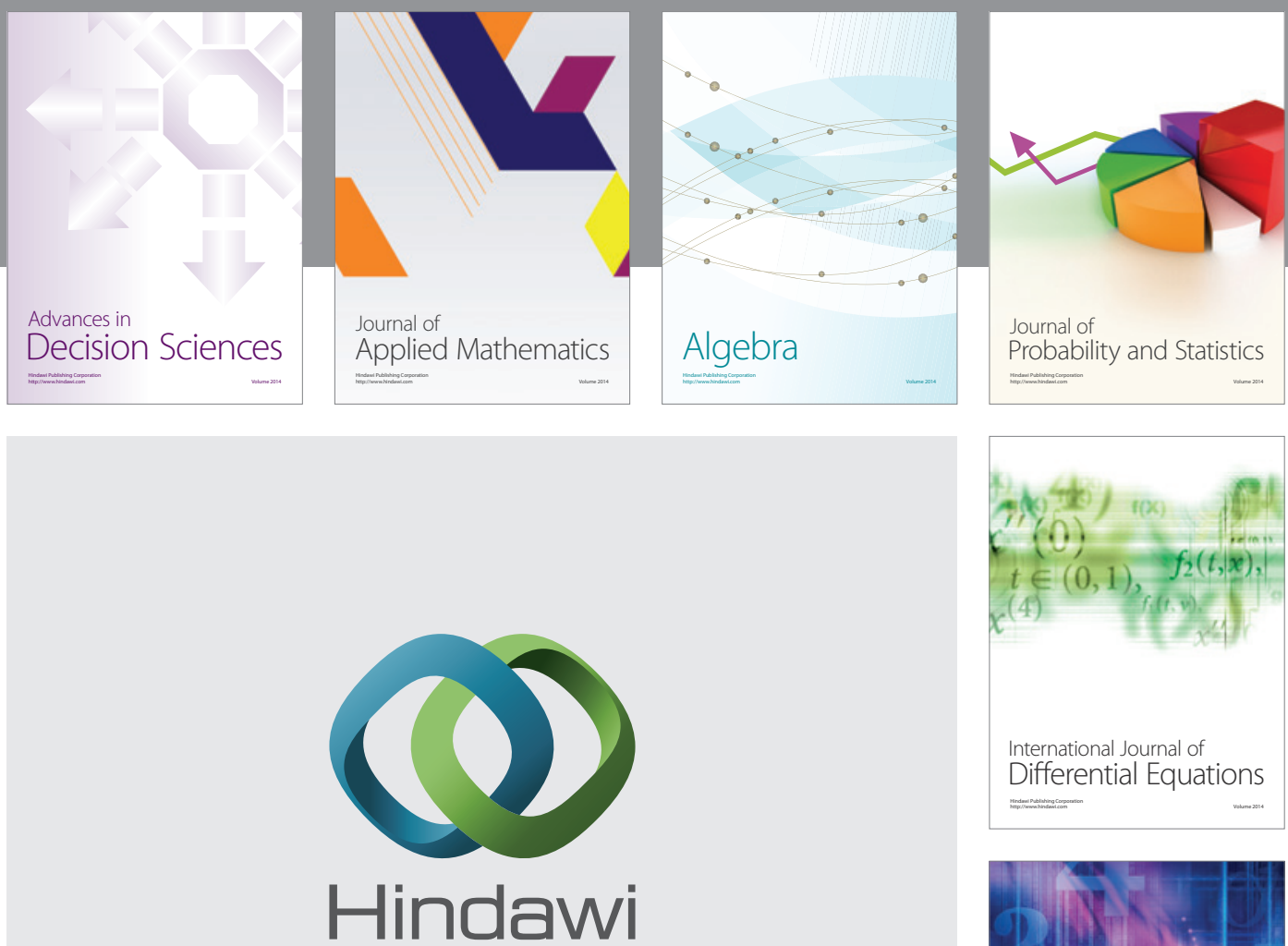

Submit your manuscripts at http://www.hindawi.com
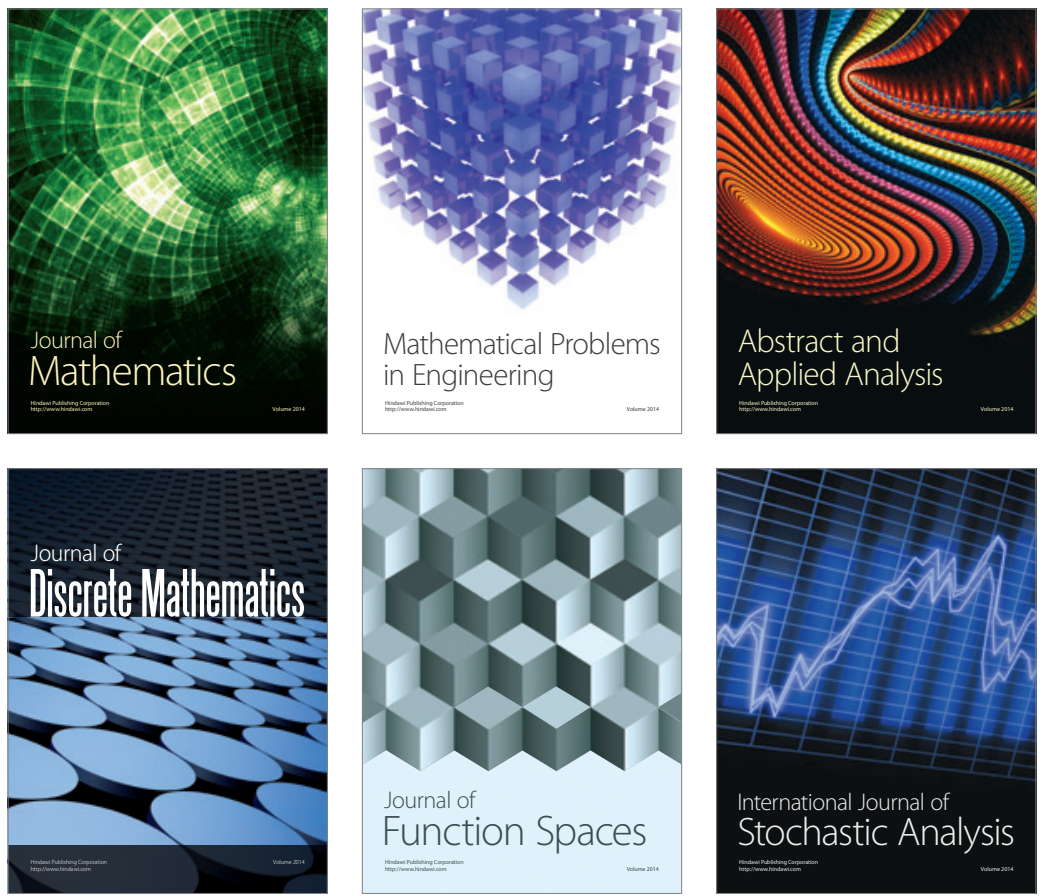

Journal of

Function Spaces

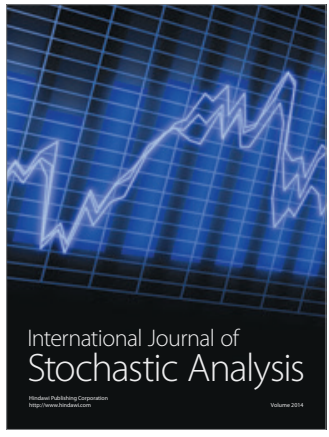

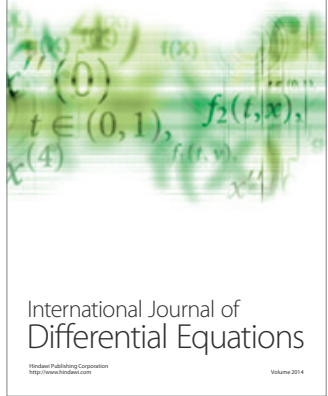
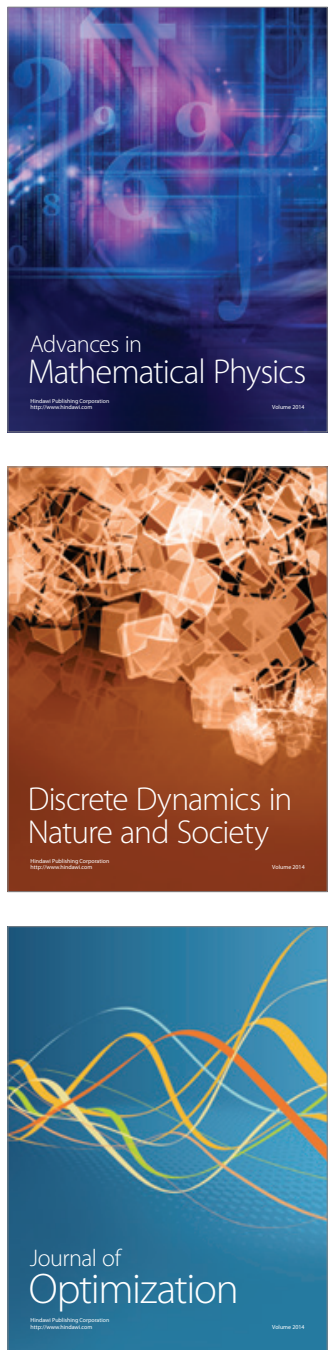